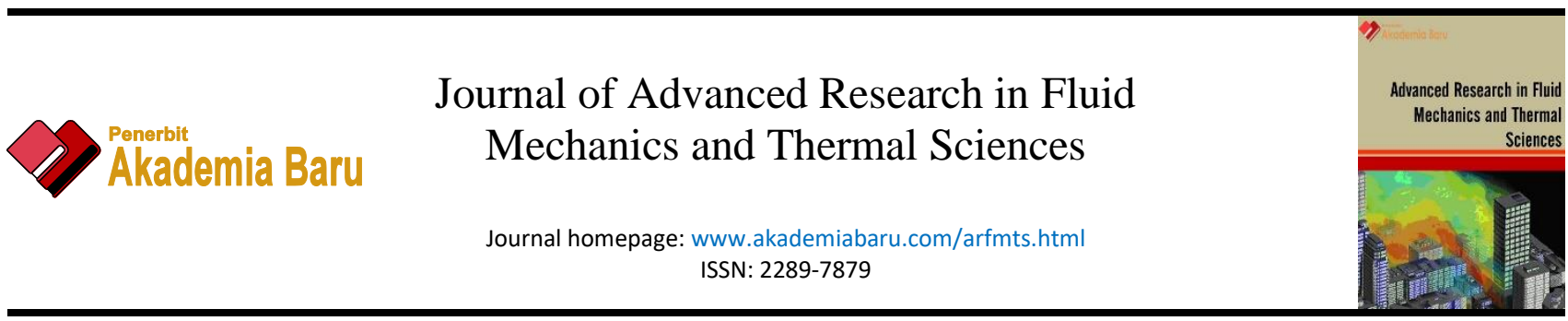

\title{
Numerical Study of the Effect of the Nanofluids Type and The Size of the Heating Sections on Heat Transfer for Cooling Electronic Components
}

\author{
Mohamed Sannad ${ }^{1,}{ }^{*}$, Btissam Abourida ${ }^{1}$, El Houcine Belarche ${ }^{1}$ \\ 1 National School of Applied Sciences, Ibn Zohr University, Agadir, BP 1136, Morocco
}

\section{ARTICLE INFO}

\section{Article history:}

Received 7 May 2020

Received in revised form 15 June 2020

Accepted 22 June 2020

Available online 15 September 2020

\section{Keywords:}

Numerical study; Natural convection; Nanofluids; Three-dimensional; SIMPLEC Algorithm

\section{ABSTRACT}

\begin{abstract}
The present work consists of analysing heat exchange by natural convection. The intensification of these exchanges and the improvement of efficiency have become a major issue in the industrial world today. This paper is part of this framework, and is particularly concerned with problems related to the intensification of heat exchanges in electronic components. Our objective is to understand the effect of the nanofluid on the mechanism of natural laminar convection in a three-dimensional cavity. In this context, we have developed our own computational code and conducted a parametric study looking at thermomechanical and geometrical parameters. The fluid flow and heat transfer in the cavity are studied for different sets of the governing parameters, namely the Rayleigh number $\mathrm{Ra}=10^{3}, 10^{4}, 10^{5}$ and $10^{6}$, volume fraction $\Phi$ varying between $\Phi=0 \%$ and $10 \%$ and nanofluid type. The obtained results show a positive effect of the volume fraction and the Rayleigh number on the heat transfer improvement. It should also be noted that the increase of the heating section size and Ra results in increased amount of heat removed by the same nanofluid. Similarly, increasing the volume fraction causes the intensification of the flow and an increase of the heat exchange.
\end{abstract}

\section{Introduction}

The flow and heat transfers induced by natural convection have been considerably studied during these last decades because of its direct application in various fields of engineering such as air conditioning, energy efficiency, cooling of electronic components, etc. The previous works had mostly focused on the improvement of heat transfer induced by natural convection, which led to the

\footnotetext{
${ }^{*}$ Corresponding author.

E-mail address: mohamedsannad@gmail.com
}

https://doi.org/10.37934/arfmts.75.2.168184 
development of a new research area based on the molecular structure of the fluid. Modern nanotechnology can produce metallic or non-metallic particles of nanometre dimensions. NanomCaterials have unique mechanical, optical, electrical, magnetic, and thermal properties. Nanofluids are engineered by suspending nanoparticles with average sizes below $100 \mathrm{~nm}$ in traditional heat transfer fluids such as water, oil, and ethylene glycol. A very small amount of guest nanoparticles, when dispersed uniformly and suspended stably in host fluids, can provide dramatic improvements in the thermal properties of host fluids.

The primary early interest in nanofluids from a technological viewpoint was the possibility of using these fluids for cooling purposes. Although the higher conductivity is an encouraging phenomenon, it is by no means conclusive evidence of the cooling capabilities of such fluids. For that, it is necessary to have definitive proof of the performance of these fluids under a convective environment. It is also important not only to reveal the convective behaviour of nanofluids but also to bring out comprehensively the fluid dynamics and heat transfer theories of nanofluids. Until now, convective studies of nanofluids have been very limited compared to experimental and theoretical studies on conduction.

Numerical work on the natural convection of nanofluids was carried out by Khanafer et al., [1]. They carried out their work on a differentially heated cavity with hot and cold vertical walls and an adiabatic horizontal wall. They showed that the rate of heat transfer increases with an increase in the volume fraction of the nanoparticles for all the considered values of the Grashof number. In addition, they have shown that the heat transfer rate is favoured when the volume fraction of the nanoparticles increases. Nnanna [2]. Experimentally examined the heat transfer behaviour of the water- $\mathrm{Al}_{2} \mathrm{O}_{3}$ nanofluid in a two-dimensional rectangular cavity with differentially heated vertical walls and adiabatic horizontal walls. He developed an empirical correlation of the Nusselt number as a function of the volume fraction of the nanoparticles and the number of Rayleighs and argued that the improved heat transfer rate can be achieved for the low values of the volume fraction of the nanoparticles.

Among the works dealing with heat transfer in the presence of nanoparticles, Murshed et al., [3]. Showed that the thermal conductivity increases with the increase of the volume concentration, and they also found that for a volume concentration of $5 \%$, the improvement of the effective thermal conductivity is about $33 \%$ for the nanotubes and of $30 \%$ for spherical nanoparticles. They then found that the size and shape of the particle have a significant influence on the thermal conductivity of nanofluids.

Similar work was carried out by Jou and Tzeng [4]. Inside a differentially heated cavity. They also used the stream function vorticity formulation in a way identical to that used in a previous study by Khanafer et al., [1]. In addition to the Grashof number effects, then described the effect of the cavity aspect ratio (width/height) on thermal behaviour. However, such results in practice because with a $20 \%$ volume fraction it is extremely difficult to make stable nanofluids difficult to use. Also, at such volume fractions, Newtonian behaviour of the fluid is doubtful. A recent numerical study by Ho et al., [5]. In a vertical square enclosure filled with a nanofluid (water- $\mathrm{Al}_{2} \mathrm{O}_{3}$ ), paints a vivid picture of the effects of modelling strategy on flow simulation. They examined the effects of uncertainties due to the adoption of different models for thermal conductivity and dynamic viscosity. This author found that the heat transfer through the enclosure could be enhanced or attenuated depending on the models used for the dynamic viscosity of the nanofluid. Hwang et al., [6]. Theoretically studied the thermal characteristics of natural convection in a rectangular cavity heated from below and filled with a water-based nanofluid containing $\mathrm{Al}_{2} \mathrm{O}_{3}$ nanoparticles. The results of the study show that the ratio of the heat transfer coefficient of the nanofluid to that of the base fluid decreases as the size of the nanoparticles increases. Hwang et al., [6]. Have made several measurements on the thermal 
conductivity of nanofluids and have shown that this parameter strongly depends on the volume fraction of the suspended particles and the thermal conductivity of the base fluid. Recently, Oztop and Abu-Nada [7]. Have numerically studied heat transfer and fluid flow as a function of buoyancy forces in a partially heated enclosure filled with nanofluids containing different types of nanoparticles. They showed that the improvement of the heat transfer was more pronounced for the low values of the aspect ratio. They found that for all Rayleigh numbers, the average Nusselt number increases as the volume fraction of the nanoparticles increases.

Anilkumar and Jilani [8]. Presented a numerical investigation on the natural convection of a mixture of ethylene glycol and aluminium oxide in an enclosure heated by an isothermal partition located on its lower wall. The study indicates the influence of the nanoparticle volume fraction, the Rayleigh number and the shape ratio of the chamber on the dynamic and thermal behaviour of the fluid. The results obtained show that the heat transfer improves with the increase of the volume fraction of the nanoparticles and the presence of the nanoparticles in the base fluid modifies the structure of the flow. Natural convection in a square enclosure inclined and heated by a heat source placed in the centre of the left wall was studied by Ögüt [9]. The cavity is filled with a mixture of water and nanoparticles $\left(\mathrm{Cu}, \mathrm{Ag}, \mathrm{CuO}, \mathrm{Al}_{2} \mathrm{O}_{3}\right.$, and $\left.\mathrm{TiO}_{2}\right)$. The results show that the type of nanoparticle is a major factor in improving heat transfer.

Garoosi et al., [10]. Conducted a numerical study on the natural convection of nanofluid in a twodimensional square cavity, the results indicated that there is an optimal volume fraction for each Rayleigh number at which the transfer rate is optimal, and they found that thermophoretic effects are negligible for nanoparticles with high thermal conductivity. Li and Nakayama [11]. Investigated the influence of the dependency of thermophysical properties of temperature on the improvement of convective heat transfer of nanofluid, they made a comparison between the solutions, with and without taking into account this dependency, the results indicated that this only reflects the relative magnitude of the Nusselt number and that it does not have a significant influence on the increase of heat transfer. Corcione [12]. Adopted the non-homogeneous model to study natural convection in a square, laterally heated enclosure filled with water- $\mathrm{Al}_{2} \mathrm{O}_{3}$ having properties depend on the temperature, found that the heat transfer performance peaks at an optimal nanoparticle volume fraction, which increases when all parameters are elevated, and developed a set of correlations underlying these results.

Sheikholeslami et al., [13] studied the magnetic field effect on CuO-water nanofluid flow and heat transfer in an enclosure which is heated from below. They found that effect of Hartmann number and heat source length is more pronounced at high Rayleigh number.

Muhammad and Sidik [14]. Studied the utilisation of Nanofluids In Minichannel For Heat Transfer and Fluid Flow Augmentation. They concluded that the following conclusions: declaration of superiority of nanofluid over base fluid on improvement of heat transfer rate, and consequently enhanced convective heat transfer coefficient (HTC), minimal pressure drops which may not necessarily demand more pumping power of working fluid and reasonable level of thermal resistance.

Mohammed et al., [15]. Examined numerically the laminar and turbulent mixed convection flows using nanofluids. These numerical results indicate that the nanofluid with $\mathrm{SiO} 2$ has the highest Nusselt number compared with other nanofluids types. The Nusselt number increases as the volume fraction of nanoparticles and the Reynolds number increase, while it decreases as the nanoparticle's diameter increases.

Mahmoudi et al., [16]. Has numerically studied natural convection in a square enclosure filled with nanofluid (Cu-water). A single heating source, generating a constant heat flow, is placed on the left vertical wall of the enclosure. The other vertical wall is maintained at a constant low temperature, 
while the other walls are thermally insulated. Guiet et al., [17]. The laminar natural convection of a mixture of water and copper nanoparticles was numerically studied by the Boltzmann method in a square cavity heated from below and cooled laterally. The heating is provided by a block, which is either kept at a constant temperature or subjected to a constant heat flow. The Brinkman [18] model and the Patel et al., [19]. Respectively calculate the effective viscosity and thermal conductivity of the nanofluid. The main parameters of the problem are the Rayleigh number, the volume fraction of the nanoparticles as well as the dimensions and position of the heating block. The results of the study show that the heat transfer improves with the increase of the volume fraction of the nanoparticles independently of the boundary conditions applied on the heating block. Flow and heat transfer are significantly affected by the size of the heating source.

Hence, the purpose of the present investigation is to study numerically the laminar natural convection in a cubical enclosure filled with nanofluid. The temperature distributions, the velocity patterns, and the heat transfer rates are analysed and discussed in this paper.

\section{Problem Formulation}

The physical model considered is shown schematically in Figure 1. It is a three-dimensional cavity of length $(\mathrm{H})$, filled with nanofluids and having two heating portions in the form of a source of length $(E)$ and height $(\varepsilon)$ on its left vertical wall.

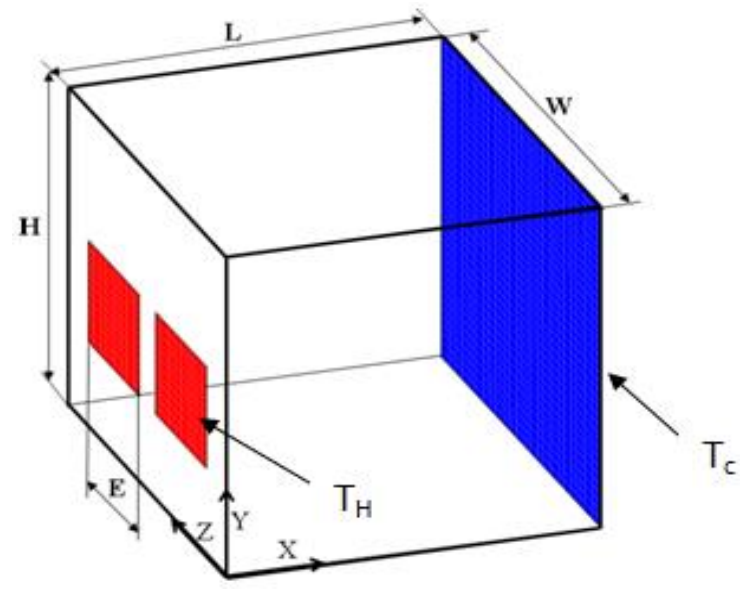

Fig. 1. Studied configurations and coordinates

The mathematical model used is based on the Navier-Stokes and energy equations. These equations are discretized by the finite volume method, taking into account the Boussinesq approximation and neglecting the viscous dissipation. The physical parameters are given by the following equations.

The density

$\rho_{n f}=(1-\phi) \rho_{f}+\phi \rho_{n p}$

The heat capacitance of the nanofluid

$$
\left(\rho C_{p}\right)=(1-\phi)\left(\rho C_{p}\right)_{f}+\phi\left(\rho C_{p}\right)_{n p}
$$


Thermal expansion coefficient

$\beta_{n f}=(1-\phi) \beta_{f}+\phi \beta_{n p}$

The dynamic viscosity of the nanofluid

Brinkman [18] has extended the Einstein formula to cover a wide range of volumetric concentrations.

$\mu_{n f}=\frac{\mu_{f}}{(1-\phi)^{2.5}}$

Thermal diffusivity of nanofluids

$\alpha_{n f}=\frac{k_{n f}}{\left(\rho C_{p}\right)_{n f}}$

The thermal conductivity of nanofluids

The formula of Maxwell [20] is given by

$\frac{k_{n f}}{k_{f}}=\frac{k_{n p}+2 k_{f}-\left[2\left(k_{f}-k_{n p}\right) \phi\right]}{k_{n p}+2 k_{f}+\left[\phi\left(k_{f}-k_{n p}\right)\right]}$

Hence, the obtained dimensionless governing equations are

$\frac{\partial U}{\partial X}+\frac{\partial U}{\partial Y}+\frac{\partial U}{\partial Z}=0$

$U \frac{\partial U}{\partial X}+V \frac{\partial U}{\partial Y}+W \frac{\partial U}{\partial Z}=-\frac{\partial P}{\partial X}+\frac{\mu_{n f}}{\alpha_{f} \rho_{n f}}\left(\frac{\partial^{2} U}{\partial X^{2}}+\frac{\partial^{2} U}{\partial Y^{2}}+\frac{\partial^{2} U}{\partial Z^{2}}\right)$

$U \frac{\partial V}{\partial X}+V \frac{\partial V}{\partial Y}+W \frac{\partial V}{\partial Z}=-\frac{\partial P}{\partial Y}+R_{a} * P_{r} * \theta * \frac{\rho_{f} \beta_{n f}}{\rho_{n f} \beta_{f}}+\frac{\mu_{n f}}{\alpha_{f} \rho_{n f}}\left(\frac{\partial^{2} V}{\partial X^{2}}+\frac{\partial^{2} V}{\partial Y^{2}}+\frac{\partial^{2} V}{\partial Z^{2}}\right)$

$U \frac{\partial W}{\partial X}+V \frac{\partial W}{\partial Y}+W \frac{\partial W}{\partial Z}=-\frac{\partial P}{\partial Z}+\frac{\mu_{n f}}{\alpha_{f} \rho_{n f}}\left(\frac{\partial^{2} W}{\partial X^{2}}+\frac{\partial^{2} W}{\partial Y^{2}}+\frac{\partial^{2} W}{\partial Z^{2}}\right)$

$U \frac{\partial \theta}{\partial X}+V \frac{\partial \theta}{\partial Y}+W \frac{\partial \theta}{\partial Z}=\frac{\alpha_{n f}}{\alpha_{f}}\left(\frac{\partial^{2} \theta}{\partial X^{2}}+\frac{\partial^{2} \theta}{\partial Y^{2}}+\frac{\partial^{2} \theta}{\partial Z^{2}}\right)$

The adopted thermal boundary conditions are

$\theta=-0.5$, on the cold wall

$\theta=0.5$, on the heated wall

$\frac{\partial \theta}{\partial n}=0$, on the adiabatic walls 
The average Nusselt number, $\mathrm{Nu}_{a}$, is defined as the integral of the temperature flux through the vertical right cold wall and formulated as

$N u_{a}=\frac{k_{n f}}{k_{f}} \int_{s} \vec{\nabla} \theta \cdot \vec{e}_{x} d y d z$

\section{Materials and Methods}

The mathematical model used is based on the equations of mass conservation, momentum conservation and energy conservation. The equations are discretized by the finite volume method using the power law scheme. The momentum conservation equations coupled with the continuity equation are solved using the SIMPLEC Algorithm. The resolution of the obtained discrete algebraic system is based on the Alternating Direction Implicit scheme (ADI). The resulting system of algebraic equations associated with the boundary conditions is solved using the Thomas Algorithm. The codes thus established have been validated by comparing them with numerical and experimental results existing in the literature.

The accuracy of the numerical code was checked by comparing our results with these previously published in the literature (Ravnik et al., [21] and Lo et al., [22]. Figure 2 and Table 1). Interesting results have been found, in terms of fluid flow and heat transfer through the cavity, depending on the adopted values of the governing parameters and also the nature of the used nanofluid.

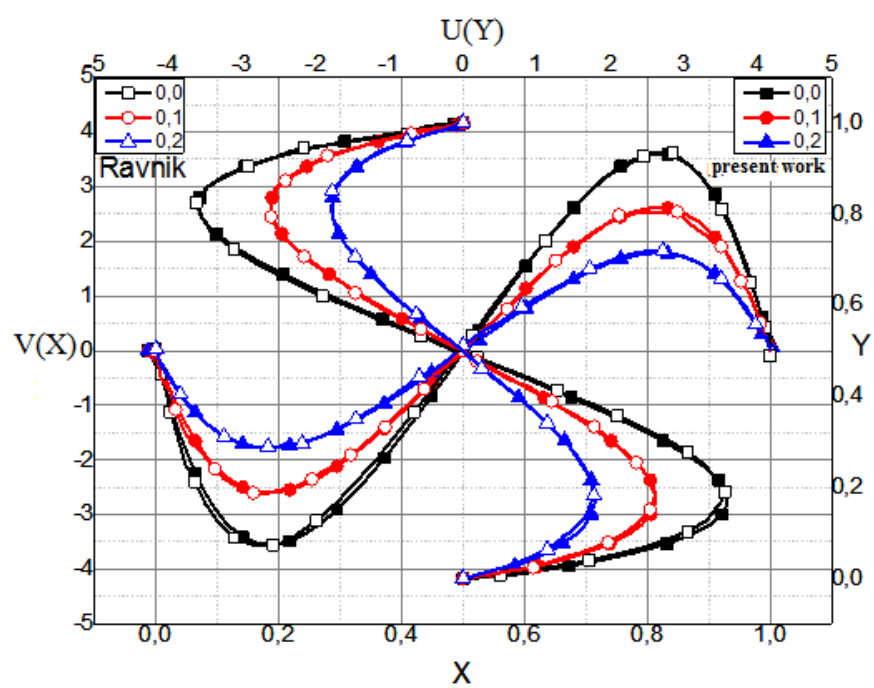

Fig. 2. Comparison of the velocity $\mathrm{V}(\mathrm{X})$ and $\mathrm{U}(\mathrm{Y})$ between our results and those of Ravnik et al., [21]

\section{Table 1}

Comparison of the average Nusselt number between our results and those of Lo et al., [22]

\begin{tabular}{llll}
\hline & Lo et al., [22] & Our study & Relative gap \\
\hline $\mathrm{Ra}=10^{3}$ & 1.0710 & 1.0770 & $0.56 \%$ \\
$\mathrm{Ra}=10^{4}$ & 2.0537 & 2.0932 & $1.9 \%$ \\
$\mathrm{Ra}=10^{5}$ & 4.3329 & 4.4329 & $2.3 \%$ \\
$\mathrm{Ra}=10^{6}$ & 8.6678 & 8.8997 & $2.6 \%$ \\
\hline
\end{tabular}




\section{Results}

The results presented in this article are obtained for Rayleigh numbers Ra ranging between $10^{3}$ and $10^{6}$ and the volume fraction equal to $0 \%$ and $6 \%$. Isotherms and streamlines are presented in order to illustrate the nanofluid motion and the heat transfer within the cavity. The variation of the average Nusselt number with the governing parameters is also investigated. The work presented is organized in two parts:

\subsection{Use of Alumina Nanoparticules $\left(\mathrm{Al}_{2} \mathrm{O}_{3}\right)$}

In this section, we consider the case where the cavity heated by two portions is filled with a waterbased nanofluid and containing nanoparticles of alumina.

\subsubsection{Flow and temperature fields}

In order to have a better view of the temperature profile within the cavity (in 3D), different planes were considered. The results obtained allowed us to choose the $Z=1 / 2$ plane as being the most representative. For the different values of $\mathrm{Ra}$, the height of each block is $\varepsilon=0.25$ and the volume fraction of nanoparticle is $\phi=4 \%$. For the low values of $\mathrm{Ra}\left(\mathrm{Ra}=10^{3}\right)$, we notice a slight distortion of the isotherms, characterizing the beginning of the appearance of the convective regime. The increase of Ra leads to a better heat transfer of the hot portions to the cold wall as shown by the corresponding isotherms. This effect is noticed for all plans considered. Due to the distribution of the imposed temperature and the boundary conditions on the vertical walls, we observed asymmetry behavior in the contour of the isotherms, which allowed us to take the plane $Z=1 / 2$ as an axis of symmetry as the Figure 3 shows. Thus, the plan chosen for the rest of our study will be that corresponding to $Z=1 / 2$ and which passes perpendicularly through the middle of component 1 . This plane suitably represents the flow and heat transfer within the cavity. The results presented are the results of simulations carried out by taking a Rayleigh number varying between $10^{3}$ and $10^{6}$ and two dimensionless height, heating portions varying from 20 to $80 \%$ of the left vertical wall.
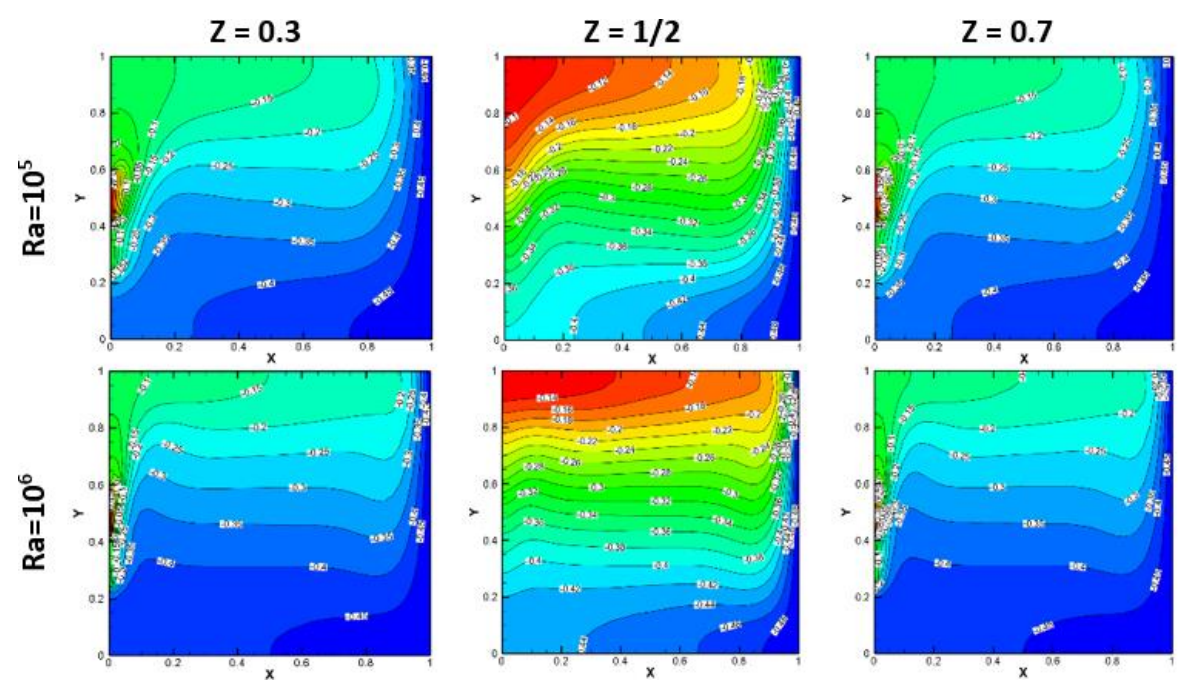

Fig. 3. Isotherms for different planes Z, different $\mathrm{Ra}, \varepsilon=0.25$ and $\phi=4 \%$ 


\subsubsection{Isotherms and streamlines}

Figure 4, Figure 5 and Figure 6 illustrate the structure of the left streamlines and the right isotherms for different values of the Rayleigh number and different dimensionless heights of the heating sources. Note that for moderate Rayleigh numbers, $\mathrm{Ra} \leq 10^{4}$, where the viscous forces are more dominant than the buoyancy forces and diffusion is the main mode of heat transfer, we have the formation of a single cell occupying the whole of the cavity whose core is located in the center and rotates clockwise. This observation is valid for all presented cases. The fluid that is heated by the left wall moves to the adiabatic upper wall, where the flow goes to the right vertical cold wall, because the density of the heated fluid decreases and so moves upwards, and that is the flow intensity of the pure fluid, which is the strongest. The temperature field is stratified along the diagonal of the cavity and the thermal gradients are weak near the active walls. For these moderate Rayleigh numbers, the effect of the dimension remains small. It should be noted that for a given Rayleigh number, the streamlines are almost identical when the dimensionless height of the heated source is varied. This result is due to the conservation of the intensity of the flow.

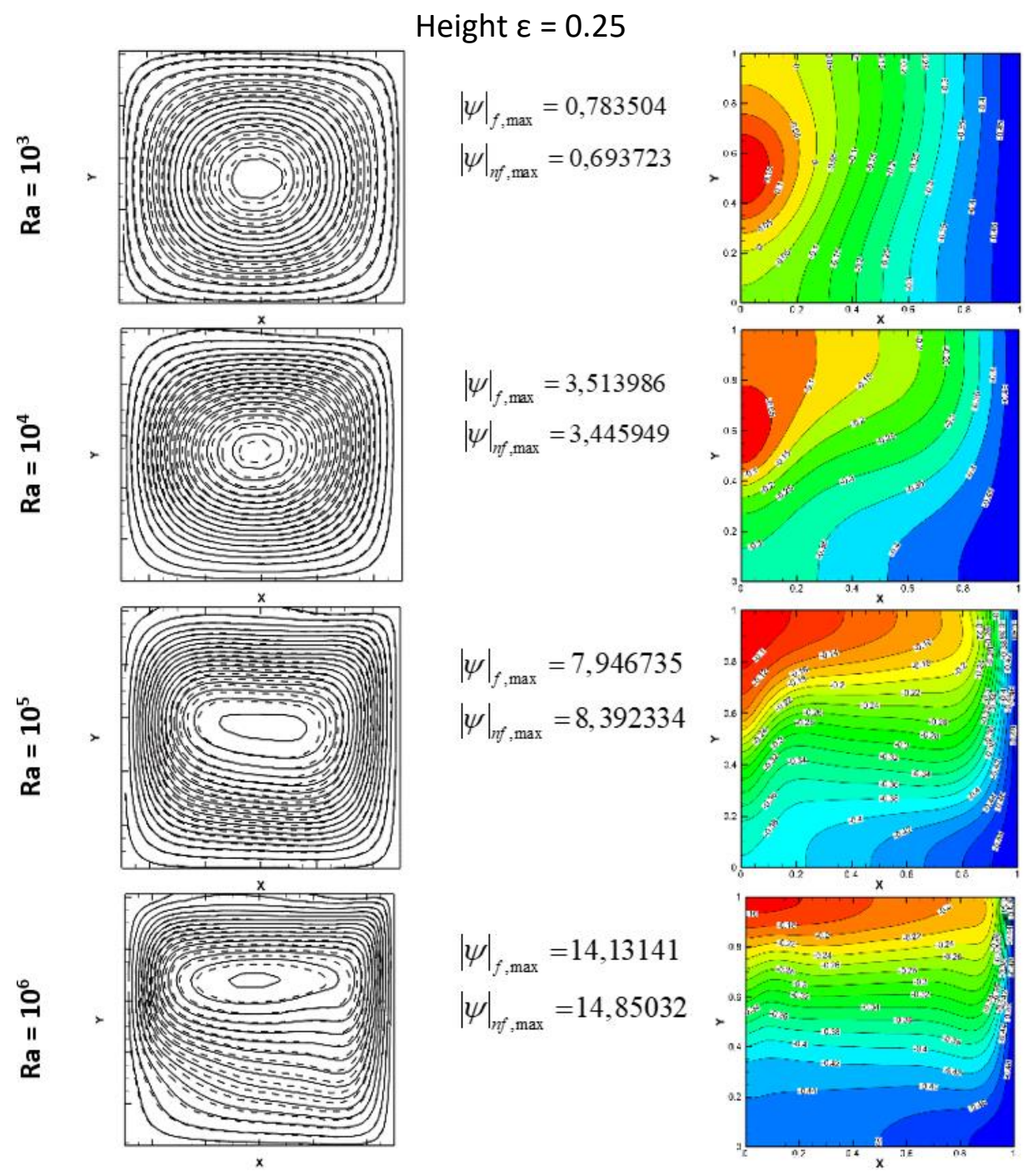

Fig. 4. Streamlines and isotherms for water and nanofluid $\left(\right.$ Water- $\left.-\mathrm{Al}_{2} \mathrm{O}_{3}\right)$ for different $\mathrm{Ra}, \phi=4 \%$ and $\varepsilon=0.25$ 
On the other hand, for a given value of the dimensionless height of the heated source, one notices that with the increase of the number of Rayleigh, the intensity of the recirculation inside the enclosure increases and the centers of the streamlines move upwards. In the case of $R a \geq 10^{5}$, the figures show that the isotherms are strongly affected by the Rayleigh number, but not as a function of the height of the source. When $\mathrm{Ra}=10^{6}$, the thermal boundary layers become thinner and the isotherms become laminated in the central region of the cavity. The increase of the stratification as a function of the dimension's height of the heated source increases for a fixed Rayleigh number. Then, we conclude that when the dimensionless height of the heated source increases, the heat transfer rate also increases, given the increase of the heat exchange surface. For a fixed value of the height of the heated source, the increase in the number of Rayleigh results in the tightening of the isotherms in the zone situated near the left wall heated by the two blocks, that is to say that the temperature gradients become more important near to the heated left wall, and the flow intensity of the nanofluid becomes stronger compared to that of the pure fluid.

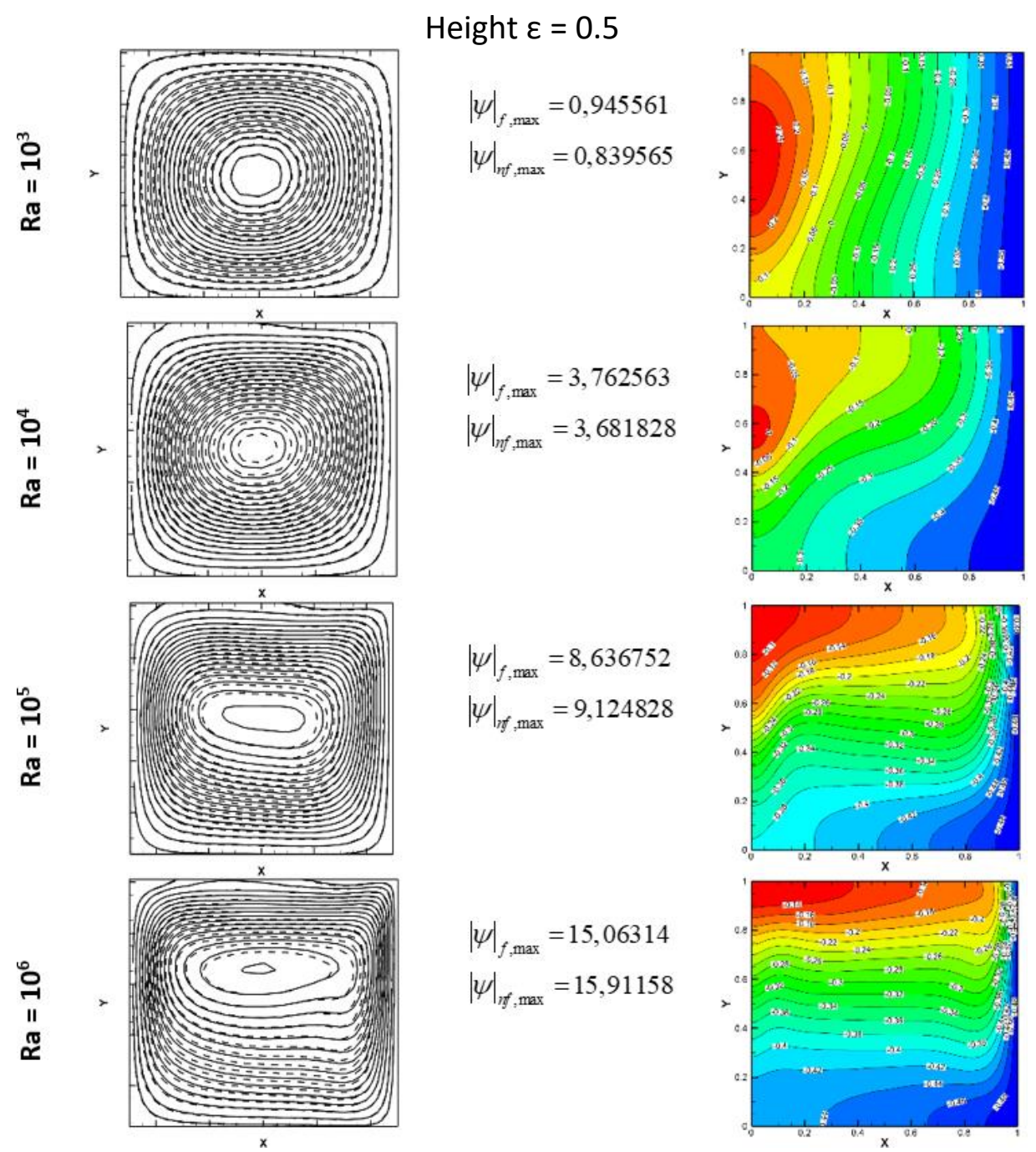

Fig. 5. Streamlines and isotherms for water and nanofluid (Water- $\left.-\mathrm{Al}_{2} \mathrm{O}_{3}\right)$ for different $\mathrm{Ra}, \phi=4 \%$ and $\varepsilon=0.5$ 


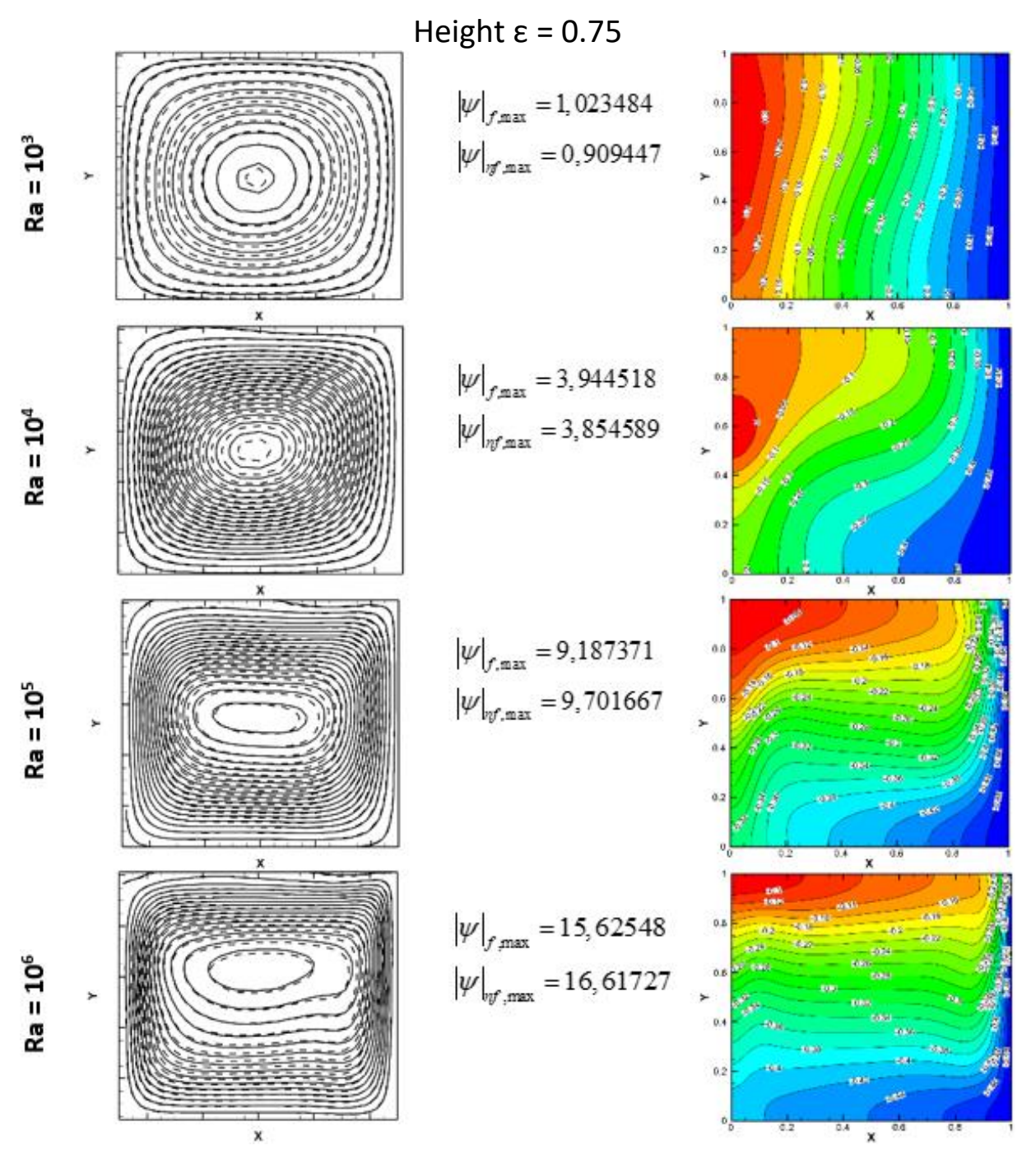

Fig. 6. Streamlines and isotherms for water and nanofluid $\left(\right.$ Water- $\left.-\mathrm{Al}_{2} \mathrm{O}_{3}\right)$ for different $\mathrm{Ra}, \phi=4 \%$ and $\varepsilon=0.75$

\subsubsection{Velocity}

In order to more justification for the flow of the fluid in the cavity, Figure 7 shows the profiles of the vertical component of the velocity $(\mathrm{V})$ along the horizontal median of the enclosure, for different heights of the heat source, and different Rayleigh numbers increases under the effect of the buoyancy force with the increase of Ra. We observe a decrease in the intensity of the fluid flow at the cold right side wall and an increase in the left side wall heated by the two blocks. This rise increases with Rayleigh number and reaches its maximum at the center of the heated wall, where the temperature is maximized. This is due to the increase in the intensity of the thermal thrust forces and therefore to the predominance of convective heat transfer. This figure thus shows the variation of the speed as a function of the height of the heat source. As the height $\varepsilon$ of the sources increases, the heat exchange surface between the fluid and the source increases. Therefore, the fluid is heated significantly, and relaxes with a decrease in density. It follows a rise of the fluid upwards and the speed of the flow becomes important. The profiles $\mathrm{V}$ show that the cell formed inside the cavity circulates clockwise, and the maximum vertical speed of the flow becomes important. 

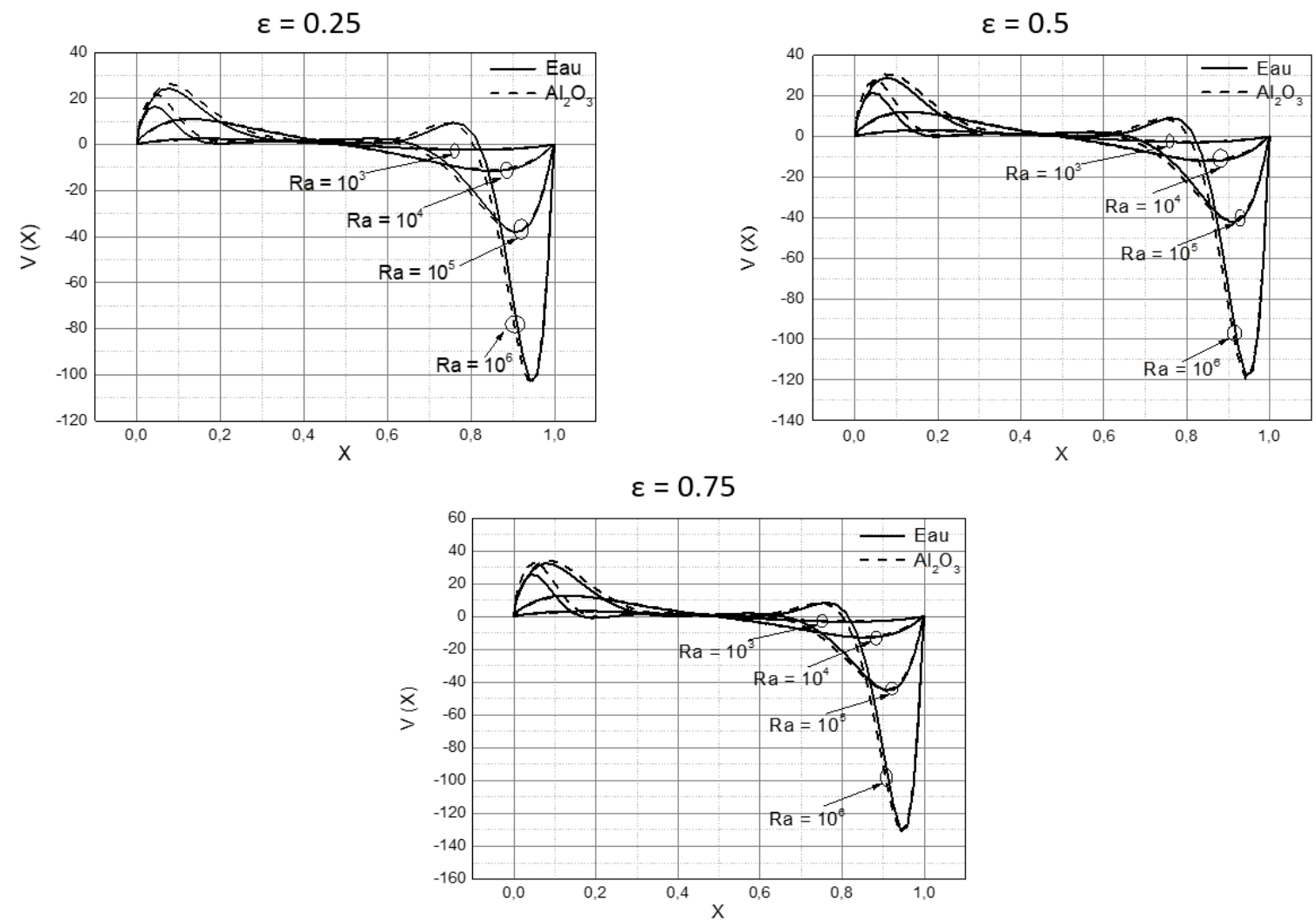

Fig. 7. Vertical component of the velocity along the horizontal median plane for pure water and nanofluid (water $+\mathrm{Al}_{2} \mathrm{O}_{3}$ ) and for different height

\subsubsection{Average Nusselt number}

The results presented in this part are obtained for Rayleigh numbers Ra between $10^{3}$ and $10^{6}$, different volume fractions of the nanoparticles, and considering the three dimensions of the heating sections $\varepsilon$. Subsequently, we will focus on the effect of these parameters on the average Nusselt number. Effect of Ra, the height of the heating surfaces $(\varepsilon)$ and the volume fraction. The variation of the average Nusselt number as a function of the height of the heat sources for different Rayleigh numbers is shown in Figure 8. It is noted that regardless of the height of the heat sources, the average Nusselt number increases with the increase of the volume fraction. This increase is due to the improvement of the thermal conductivity of the nanofluid. For a given height $\varepsilon$, the average Nusselt number increases with increasing Rayleigh number. It can also be seen that to a height of the heat source $\varepsilon=0.75$, the Nusselt number reaches the maximum value of all the Rayleigh numbers considered. Indeed, as shown in Figure 6, the isotherms that correspond to this dimension, show strong thermal gradients on all sides of the heat source, compared to other heights. 

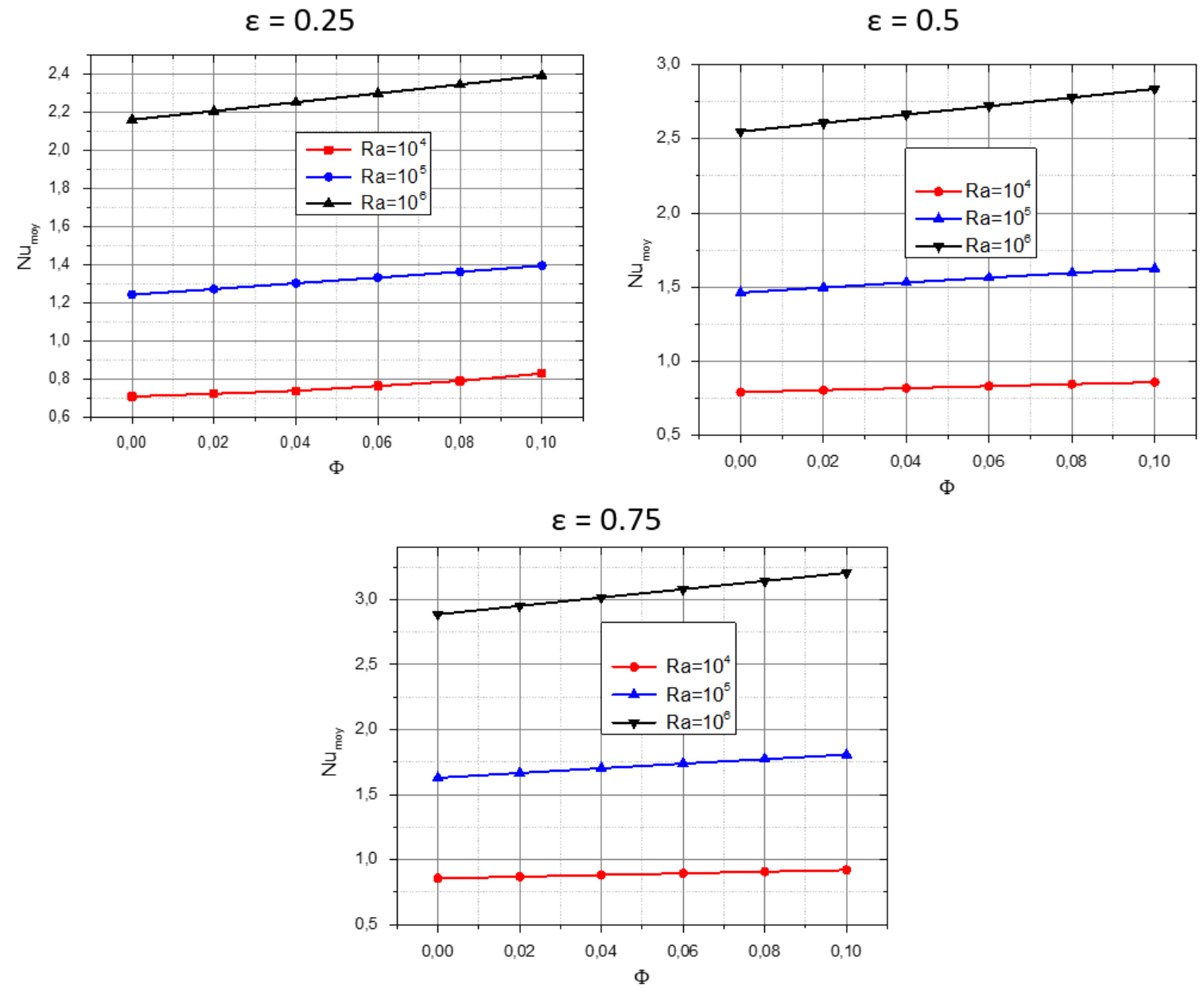

Fig. 8. Variation of the average Nusselt number as a function of the volume fraction for different Ra and $\varepsilon$

\subsection{Thermal Comparison Between Different Types of The Nanoparticles}

We study in this part, the influence of different types of nanoparticles $\left(\mathrm{Al}_{2} \mathrm{O}_{3}, \mathrm{Cu}\right.$ and $\left.\mathrm{TiO}_{2}\right)$ dispersed in the base fluid on the heat transfer. Numerical simulations are performed for adimensional height of each portion fixed at $\varepsilon=0.5$, different volume fraction and $\mathrm{Ra}$. The comparisons conducted between the three types of nanoparticles, concern primarily the dynamic and thermal fields, then the heat transfer.

\subsubsection{Isotherms and streamlines}

Figure 9 illustrate the isotherms and the streamlines for different types of nanoparticles $\left(\mathrm{Al}_{2} \mathrm{O}_{3}\right.$, $\mathrm{Cu}$ and $\mathrm{TiO}_{2}$ ) and a pure fluid in the $\mathrm{Z}=1 / 2$ plane, the volume fractions varying between $0 \%$ and $4 \%$ and the Rayleigh number ( $\mathrm{Ra}=10^{4}, 10^{5}$ and $\left.10^{6}\right)$, with a heat source of fixed height $\varepsilon=0.5$. The comparison between the nanofluids and the pure fluid shows a significant deviation of the isotherms by increasing the Rayleigh number and the volume fraction. For $\mathrm{Ra}=10^{3}$, these isotherms are nearly parallel to the vertical walls, indicating that for small values of the Rayleigh number, the heat transfer is practically conductive. For $\mathrm{Ra}=10^{5}$, the isotherms are slightly deformed with a small variation following $\mathrm{y}$. Beyond this value of Ra, the deformation of the isotherms becomes more evident and horizontal stratification begins to settle in the center of the cavity. For $\mathrm{Ra}=10^{6}$, we notice that the vertical gradient of the temperature becomes almost constant at the heart of the cavity and 
subsequently a horizontal stratification settles there. The values of $\psi$ (the function of the current) increase with the increase of Rayleigh number. Thus, it is shown that the flow of the pure fluid is stronger than that of the nanofluid for $\mathrm{Ra}=10^{3}$. The streamlines become tighter close to the sidewalls, and the shape of the cells changes completely for $\mathrm{Ra} \geq 10^{5}$. The flow of the nanofluids becomes stronger. According to this figure, the water and the different mixtures have the same dynamic and thermal behavior. Furthermore, Figure 9, confirms that the copper nanoparticles allow a better heat exchange by comparing with Alumina and Titanium and the type of nanoparticles is a main factor for the improvement of the heat transfer.

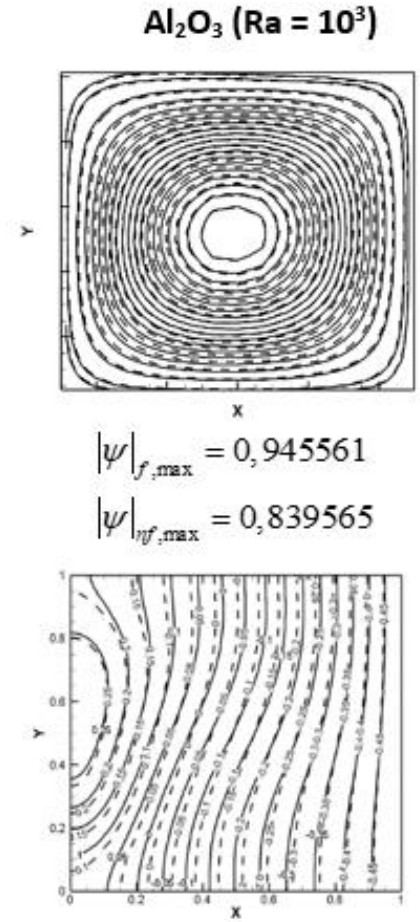

$\mathrm{Al}_{2} \mathrm{O}_{3}\left(\mathrm{Ra}=10^{6}\right)$
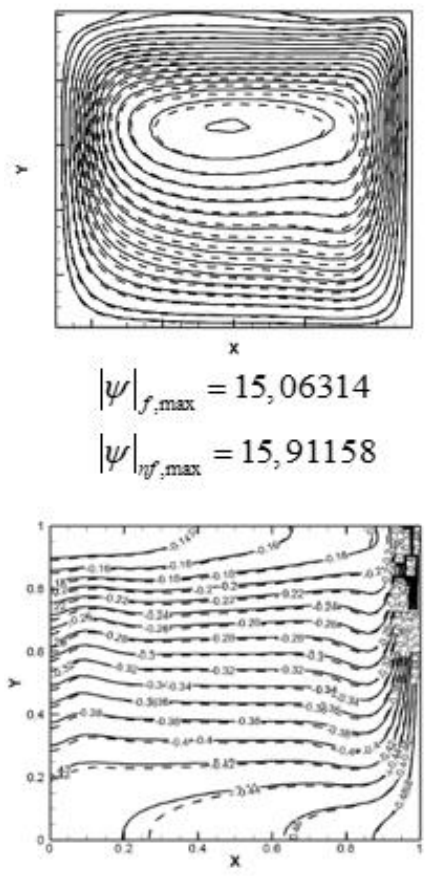

$\mathrm{Cu}\left(\mathrm{Ra}=10^{3}\right)$

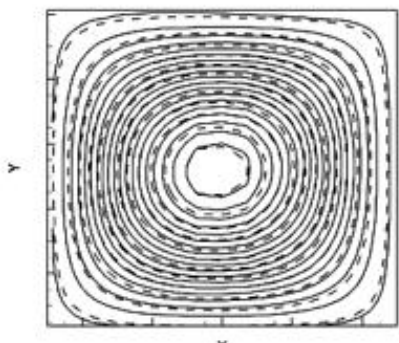

$|\psi|_{f, \max }=0,945561$

$|\psi|_{n f, \max }=0,848565$

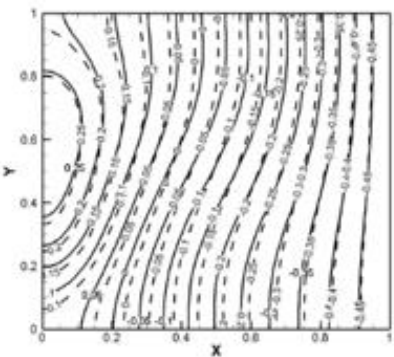

$\mathrm{Cu}\left(\mathrm{Ra}=10^{6}\right)$

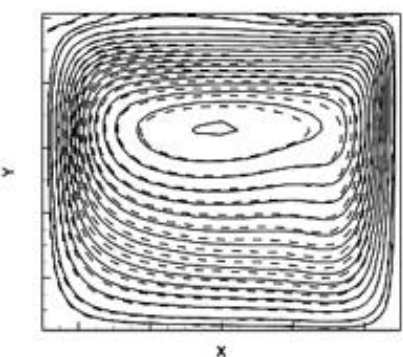

$|\psi|_{f \max }=15,06314$

$|\psi|_{\text {, } \max }=15,92350$

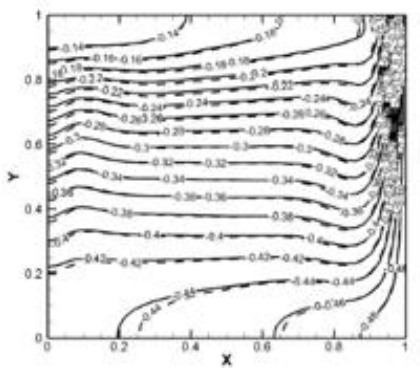

$\mathrm{TiO}_{2}\left(\mathrm{Ra}=10^{3}\right)$

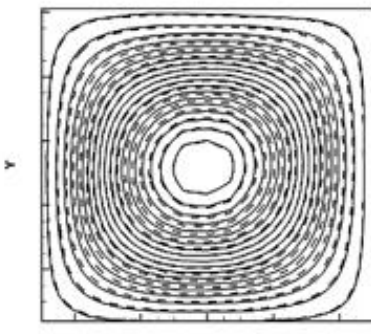

$|\psi|_{f, \text { max }}=0,945561$

$|\psi|_{n f \text { max }}=0,839611$

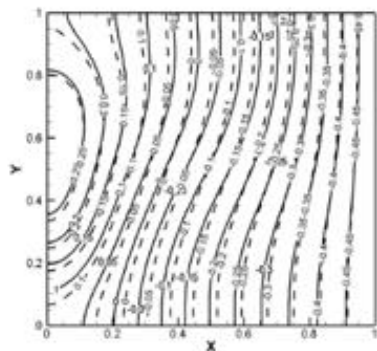

$\mathrm{TiO}_{2}\left(\mathrm{Ra}=10^{6}\right)$

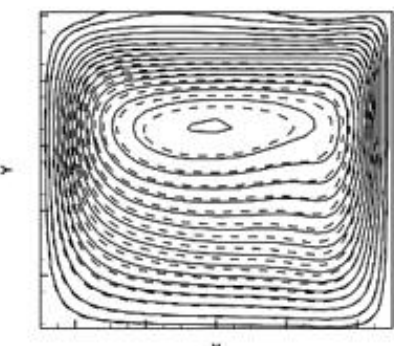

$|\psi|_{f \max }=15,06314$

$|\psi|_{f_{f, \text { max }}}=15,74973$

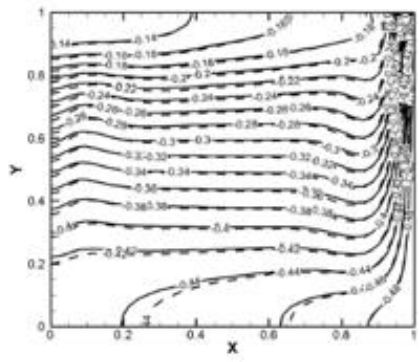

Fig. 9. Streamlines (top) and isotherms (bottom) for pure water (-) and for different nanofluids (----) in the plane $Z=1 / 2$ for $\varepsilon=0.5$ and $R a=10^{3}$ and $10^{6}$ 


\subsubsection{Velocity}

Figure 10 shows the vertical component velocity profiles at the center line for different Rayleigh number values ( $\mathrm{Ra}=10^{3}, 10^{4}, 10^{5}$ and $\left.10^{6}\right)$. From this figure, we notice that the vertical velocity $(\mathrm{V})$ reaches a maximum value near to the adiabatic sidewalls, for all types of nanoparticles, where the fluid changes direction and $V$ decreases towards negative values. The difference between the three types of nanofluids is small and the vertical component of the velocity is not significantly affected by the type of nanoparticles.
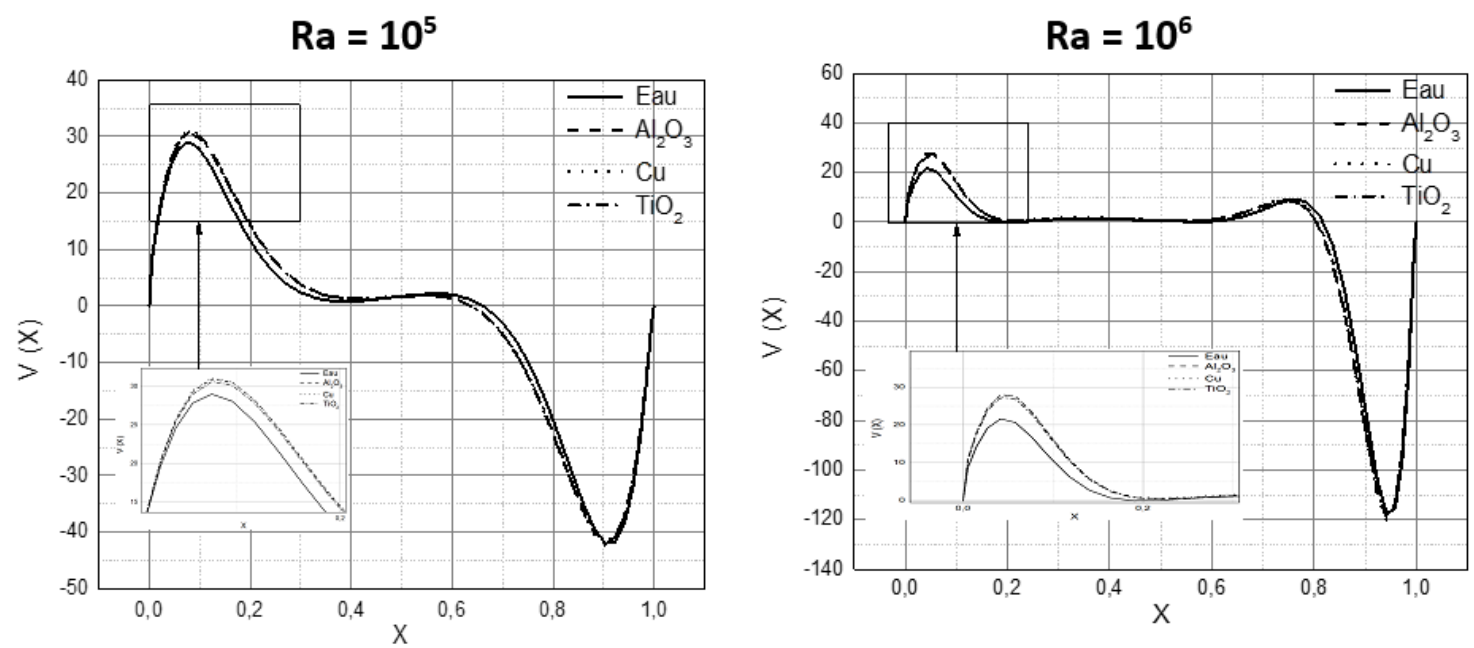

Fig. 10. Vertical component of velocity at $Z=1 / 2$ for pure water and different nanofluids with $\phi=4 \%$ and $\varepsilon=0.5$

\subsubsection{Average Nusselt number}

The variation of the average Nusselt number along the cold wall is illustrated in Figure 11, for different nanoparticles, $\mathrm{Ra} \geq 10^{4}$ and $\varepsilon=0.5$. The figure shows that the number of Nusselt increases with the increasing Rayleigh number and height of the heat source for different types of nanoparticles. As for the influence of the nanoparticle type on heat transfer, the figure further confirms that the copper-based nanofluid provides the best heat transfer, followed by Aluminum Oxide and Titanium Oxide because of the importance of its thermal conductivity compared to the other nanoparticles. 

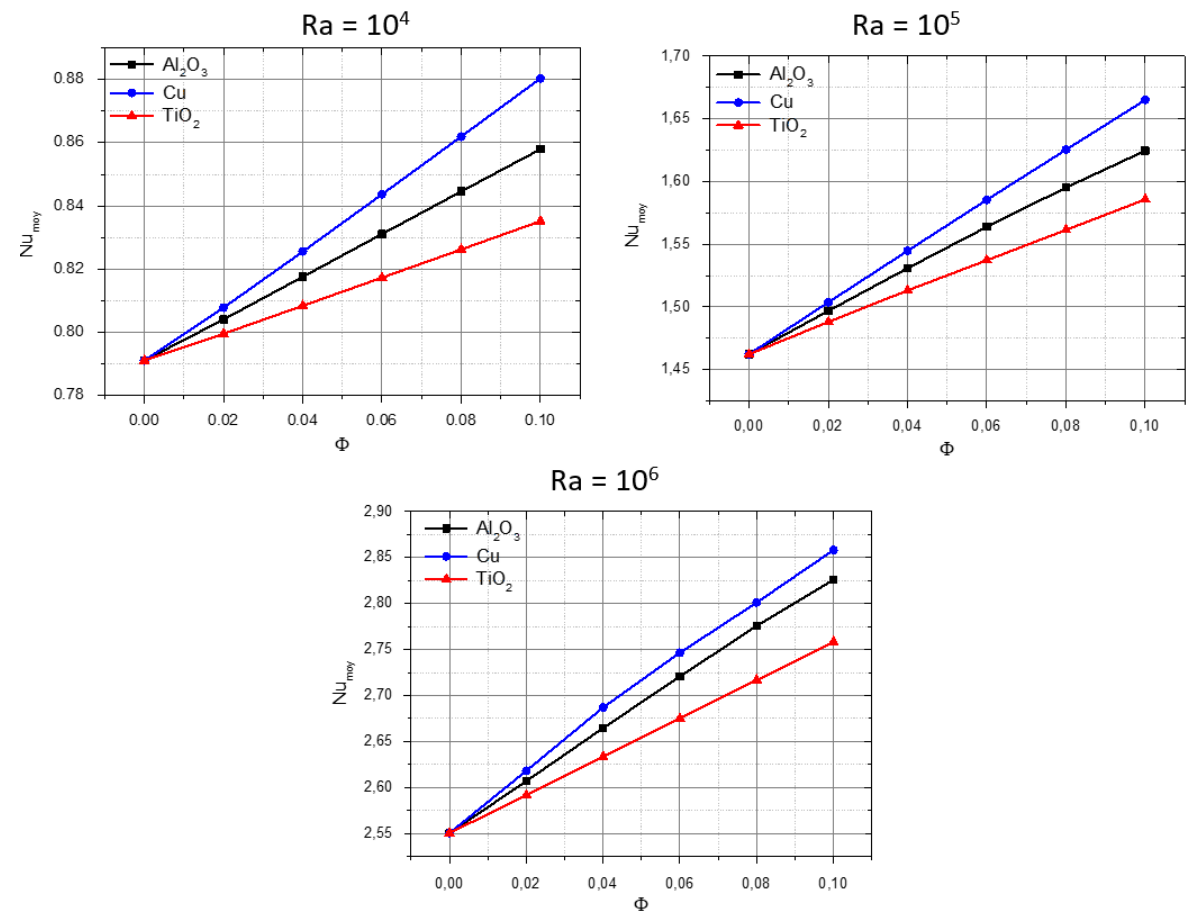

Fig. 11. Variation of average Nusselt number as a function of $\phi$ for different types of nanoparticles, different Rayleigh and for $\varepsilon=0.5$

\section{Conclusions}

The study presented in this chapter deals with the natural laminar convection of nanofluids within a three-dimensional cavity containing two heating portions located on the left wall. In the first part of this paper, we analyzed the effect of the various parameters on heat transfer, namely the volume fraction, the Rayleigh number and the height of the heat source. This allowed us to highlight the effect generated by each parameter and to identify the optimal situations favouring heat transfer. In the second part, we made a comparison between three types of nanoparticles (Copper (Cu), Aluminum Oxide $\left(\mathrm{Al}_{2} \mathrm{O}_{3}\right)$ and Titanium Oxide $\left.\left(\mathrm{TiO}_{2}\right)\right)$. The analysis of the results related to the heat transfer shows that the copper-based nanofluid guarantees the best thermal transfer in the considered case. It should also be noted that the increase of the heating section size and Ra results in increased amount of heat removed by the same nanofluid. Similarly, increasing the volume fraction causes the intensification of the flow and an increase of the heat exchange.

\section{Acknowledgement}

This research was not funded by any grant.

\section{References}

[1] Khanafer, Khalil, Kambiz Vafai, and Marilyn Lightstone. "Buoyancy-driven heat transfer enhancement in a twodimensional enclosure utilizing nanofluids." International Journal of Heat and Mass Transfer 46, no. 19 (2003): 3639-3653.

https://doi.org/10.1016/S0017-9310(03)00156-X

[2] Nnanna, A. G. Agwu. "Experimental model of temperature-driven nanofluid." Journal of Heat Transfer 129, no. 6 (2007): 697-704. https://doi.org/10.1115/1.2717239

[3] Murshed, S. M. S., K. C. Leong, and C. Yang. "Enhanced thermal conductivity of TiO2-water based nanofluids." International Journal of Thermal Sciences 44, no. 4 (2005): 367-373.

https://doi.org/10.1016/j.ijthermalsci.2004.12.005 
[4] Jou, Rong-Yuan, and Sheng-Chung Tzeng. "Numerical research of nature convective heat transfer enhancement filled with nanofluids in rectangular enclosures." International Communications in Heat and Mass Transfer 33, no. 6 (2006): 727-736. https://doi.org/10.1016/j.icheatmasstransfer.2006.02.016

[5] Ho, Ching-Jenq, M. W. Chen, and Z. W. Li. "Numerical simulation of natural convection of nanofluid in a square enclosure: effects due to uncertainties of viscosity and thermal conductivity." International Journal of Heat and Mass Transfer 51, no. 17-18 (2008): 4506-4516.

https://doi.org/10.1016/i.ijheatmasstransfer.2007.12.019

[6] Hwang, Y., H. S. Park, J. K. Lee, and W. H. Jung. "Thermal conductivity and lubrication characteristics of nanofluids." Current Applied Physics 6 (2006): e67-e71.

https://doi.org/10.1016/i.cap.2006.01.014

[7] Oztop, Hakan F., and Eiyad Abu-Nada. "Numerical study of natural convection in partially heated rectangular enclosures filled with nanofluids." International Journal of Heat and Fluid Flow 29, no. 5 (2008): 1326-1336. https://doi.org/10.1016/j.ijheatfluidflow.2008.04.009

[8] Anilkumar, S. H., and Ghulam Jilani. "Convective heat transfer enhancement in an enclosure with fin utilizing nanofluids." International Journal of Physical and Mathematical Sciences 3, no. 2 (2009): 104-111.

[9] Öğüt, Elif Büyük. "Natural convection of water-based nanofluids in an inclined enclosure with a heat source." International Journal of Thermal Sciences 48, no. 11 (2009): 2063-2073.

https://doi.org/10.1016/i.ijthermalsci.2009.03.014

[10] Garoosi, Faroogh, Gholamhossein Bagheri, and Mohammad Mehdi Rashidi. "Two phase simulation of natural convection and mixed convection of the nanofluid in a square cavity." Powder Technology 275 (2015): 239-256. https://doi.org/10.1016/i.powtec.2015.02.013

[11] Li, Wenhao, and Akira Nakayama. "Temperature dependency of thermophysical properties in convective heat transfer enhancement in nanofluids." Journal of Thermophysics and Heat Transfer 29, no. 3 (2015): 504-512. https://doi.org/10.2514/1.T4460

[12] Corcione, Massimo. "Empirical correlating equations for predicting the effective thermal conductivity and dynamic viscosity of nanofluids." Energy Conversion and Management 52, no. 1 (2011): 789-793. https://doi.org/10.1016/i.enconman.2010.06.072

[13] Sheikholeslami, M., M. Gorji-Bandpy, D. D. Ganji, and Soheil Soleimani. "Natural convection heat transfer in a cavity with sinusoidal wall filled with CuO-water nanofluid in presence of magnetic field." Journal of the Taiwan Institute of Chemical Engineers 45, no. 1 (2014): 40-49.

https://doi.org/10.1016/j.jtice.2013.04.019

[14] Muhammad, Nura Mu'az, and Nor Azwadi Che Sidik. "Utilisation of Nanofluids In Minichannel For Heat Transfer and Fluid Flow Augmentation." Journal of Advanced Research Design 50, no. 1 (2018): 18-45.

[15] Mohammed, H. A., O. A. Alawi, and N. A. Che Sidik. "Mixed convective nanofluids flow in a channel having forwardfacing step with baffle." Journal of Advanced Research in Applied Mechanics 24, no. 1 (2016): 1-21.

[16] Mahmoudi, Amir Houshang, Mina Shahi, Abbas Honarbakhsh Raouf, and Ali Ghasemian. "Numerical study of natural convection cooling of horizontal heat source mounted in a square cavity filled with nanofluid." International Communications in Heat and Mass Transfer 37, no. 8 (2010): 1135-1141. https://doi.org/10.1016/i.icheatmasstransfer.2010.06.005

[17] Guiet, J., M. Reggio, and P. Vasseur. "Natural convection of nanofluids in a square enclosure with a protruding heater." Advances in Mechanical Engineering 4 (2012): 167296.

https://doi.org/10.1155/2012/167296

[18] Brinkman, H. C. "The viscosity of concentrated suspensions and solutions." The Journal of Chemical Physics 20, no. 4 (1952): 571-571. https://doi.org/10.1063/1.1700493

[19] Patel, Hrishikesh E., K. B. Anoop, Thirumalachari Sundararajan, and Sarit Kumar Das. "A micro-convection model for thermal conductivity of nanofluids." In International Heat Transfer Conference 13. Begel House Inc., 2006. https://doi.org/10.1615/IHTC13.p8.240

[20] Maxwell, James Clerk. A Treatise on Electricity and Magnetism. Vol. 1. Clarendon press, 1873.

[21] Ravnik, J., L. Škerget, and M. Hriberšek. "Analysis of three-dimensional natural convection of nanofluids by BEM." Engineering Analysis with Boundary Elements 34, no. 12 (2010): 1018-1030.

https://doi.org/10.1016/i.enganabound.2010.06.019

[22] Lo, D. C., D. L. Young, K. Murugesan, C. C. Tsai, and M. H. Gou. "Velocity-vorticity formulation for 3D natural convection in an inclined cavity by DQ method." International Journal of Heat and Mass Transfer 50, no. 3-4 (2007): 479-491.

https://doi.org/10.1016/i.ijheatmasstransfer.2006.07.025 
[23] Patankar, Suhas. Numerical Heat Transfer and Fluid Flow. Hemisphere Publishing Corporation, 1980.

[24] Patankar, Suhas V., and Dudley Brian Spalding. "A calculation procedure for heat, mass and momentum transfer in three-dimensional parabolic flows." International Journal of Heat and Mass Transfer 15, no. 10 (1972): 1787-1806. https://doi.org/10.1016/0017-9310(72)90054-3 